\title{
Infections with Carbapenem-Resistant Gram-Negative Bacteria are a Serious Problem Among Critically Ill Children: A Single-Centre Retrospective Study
}

\author{
Fatih Aygun 1,* , Fatma Deniz Aygun ${ }^{2}$, Fatih Varol ${ }^{1}$, Cansu Durak ${ }^{1}$, Haluk Çokuğraş ${ }^{2}$, \\ Yıldız Camcıoğlu ${ }^{2}$ and Halit Çam ${ }^{1}$ \\ 1 Department of Pediatric Intensive Care Unit, Istanbul University Cerrahpasa Medical Faculty, Fatih, \\ Istanbul 34098, Turkey; dr_fvarol@yahoo.com (F.V.); bzmrt@hotmail.com (C.D.); \\ hacam@istanbul.edu.tr (H.Ç.) \\ 2 Department of Infectious Disease, Istanbul University Cerrahpasa Medical Faculty, Fatih, Istanbul 34098, \\ Turkey; fdenizaygun@gmail.com (F.D.A.); cokugras@gmail.com (H.Ç.); camciy@yahoo.com (Y.C.) \\ * Correspondence: faygun999@gmail.com; Tel.: +90-(532)532-786-86-82; +90-(530)5534513; \\ Fax: +90-(212)-6328633
}

Received: 23 April 2019; Accepted: 20 May 2019; Published: 21 May 2019

\begin{abstract}
Children in paediatric intensive care units (PICUs) are vulnerable to infections because invasive devices are frequently used during their admission. We aimed to determine the prevalence, associated factors, and prognosis of infections in our PICU. This retrospective study evaluated culture results from 477 paediatric patients who were treated in the PICU between January 2014 and March 2019. Ninety patients (18.9\%) had bacterial infections, with gram-negative bacteria being the predominant infectious agents. Culture-positive patients were younger than culture-negative patients, and age was related to mortality and various clinical factors. Culture-positive bacterial infections in the PICU were associated with increased use of invasive mechanical ventilation (odds ratio(OR); 2.254), red blood cell (RBC) transfusions (OR:2.624), and inotropic drugs (OR:2.262). Carbapenem resistance was found in approximately one-third of gram-negative bacteria, and was most common in tracheal aspirate specimens and cases involving Klebsiella spp. Total parenteral nutrition was a significant risk factor (OR:5.870). Positive blood culture results were associated with poorer patient survival than other culture results. These findings indicate that infections, especially those involving carbapenem-resistant bacteria, are an important issue when treating critically ill children.
\end{abstract}

Keywords: carbapenem resistance; paediatric patients; nosocomial infection; colistin; tigecycline

\section{Introduction}

Infections are a common cause of mortality in the paediatric intensive care unit (PICU) [1-3], with mortality rates of up to 50\% depending on the infection's origin [1]. Infants and young children have especially high risks, based on the immaturity of their immune system, their lack of prior exposure to antigens, and their physical barriers' relative permeability to microorganisms [3]. In addition, children in the PICU are vulnerable to infections because invasive devices are frequently used during their hospitalization [2]. Therefore, the incidence of nosocomial infections in the PICU is higher than in other wards [4]. Nosocomial infections are becoming increasingly common in intensive care units, with higher rates of carbapenem-resistant bacterial infections (CRI), which has led to increased use of colistin and other last resort antibiotics [5]. In addition, MDR infections in children, particularly CRI in the PICU, are poorly described in the literature [6]. Therefore, early infection detection is critical in the PICU, and we aimed to determine the prevalence, risk factors, and prognosis of infections in our PICU. 


\section{Results}

\subsection{Demographic Characteristics}

Between January 2014 and March 2019, 513 children were admitted to our PICU and 477 patients were considered eligible for the present study (Figure 1). Thirty-six patients were excluded because of a PICU stay duration of $<24 \mathrm{~h}$ or death within the first $24 \mathrm{~h}$ after hospitalization (17 cases) or fungal and multi-bacterial culture results (19 cases).

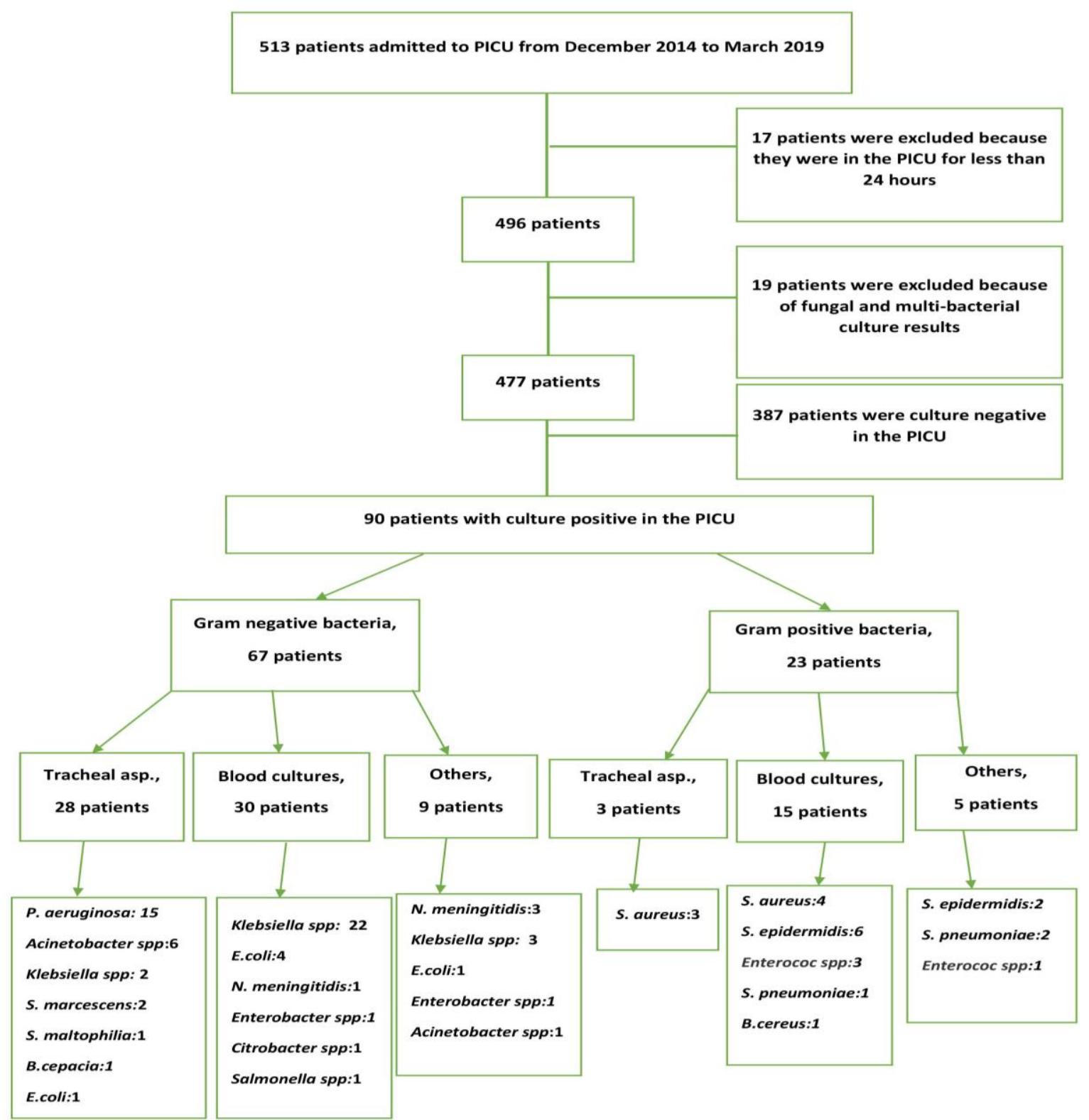

Figure 1. Study flow chart.

The demographic characteristics of the 477 included patients are shown in Table 1 . The patients included 265 males (55.6\%) and 212 females (44.4\%), with age ranging from 1 day to 18 years (median age: 2.0 years). The most frequent causes of PICU admission were respiratory disorders (188 patients; $24.7 \%$ ), metabolic diseases (85 patients; $17.8 \%$ ), sepsis (61 patients; $12.8 \%$ ), surgery (57 patients; $11.9 \%$ ), and neurological diseases (50 patients; $10.5 \%$ ). The median PICU stay duration was 3.0 days (range: 2-122 days). Inotropic drugs were used for 103 patients (21.6\%). Invasive mechanical 
ventilation (IMV) was used for 194 patients (40.7\%) and non-invasive mechanical ventilation (NIV) was used for 161 patients (33.8\%). Continuous renal replacement therapy (CRRT) was performed for 100 patients $(21.0 \%)$. Thirty-seven patients $(7.8 \%)$ died during their PICU stay. The central catheter-related bloodstream infection rate was 2.12/1000 catheter-days and the ventilator-associated pneumonia rate was 4.98/1000 ventilator-days.

Table 1. Demographic characteristics of the 477 patients in the paediatric intensive care unit between January 2014 and March 2019.

\begin{tabular}{|c|c|}
\hline Sex & \\
\hline Male & $265(55.6 \%)$ \\
\hline Female & $212(44.4 \%)$ \\
\hline \multicolumn{2}{|l|}{ Reason for Hospitalisation } \\
\hline Respiratory disease & $118(24.7 \%)$ \\
\hline Metabolic disease & $85(17.8 \%)$ \\
\hline Sepsis & $61(12.8 \%)$ \\
\hline Surgery & $57(11.9 \%)$ \\
\hline Neurologic disease & $50(10.5 \%)$ \\
\hline Haematology-oncology & $30(6.3 \%)$ \\
\hline Nephrology & $28(5.9 \%)$ \\
\hline Cardiovascular disease & $16(3.4 \%)$ \\
\hline Other & $32(7.2 \%)$ \\
\hline Age & 2.0 years ( 1 day to 18 years) \\
\hline Acute kidney injury & $137(28.7 \%)$ \\
\hline Inotropic medication & $103(21.6 \%)$ \\
\hline Continuous renal replacement therapy & $100(21.0 \%)$ \\
\hline Invasive mechanical ventilation & $194(40.7 \%)$ \\
\hline Duration of PICU stay (days) & $3.00(2-122)$ \\
\hline Central venous catheter & $251(52.6 \%)$ \\
\hline Red blood cell transfusion & $216(45.3 \%)$ \\
\hline Non-invasive mechanical ventilation & $161(33.8 \%)$ \\
\hline Mortality & $37(7.8 \%)$ \\
\hline Intravenous immunoglobulin use & $55(11.5 \%)$ \\
\hline Infections detected at PICU admission & $71(14.9 \%)$ \\
\hline Nosocomial infection in the PICU & $19(4.0 \%)$ \\
\hline Total parenteral nutrition use & $126(26.4 \%)$ \\
\hline Previous hospitalization & $250(52.4 \%)$ \\
\hline
\end{tabular}

Values are reported as number (percentage) or median (range). PICU, paediatric intensive care unit.

\subsection{Factors Associated with Culture-Positive Bacterial Infections in the PICU}

There were relationships between culture-positive bacterial infections and patient age, IMV support, duration of IMV use, inotropic drug use, acute kidney injury (AKI), NIV support, mortality, duration of NIV use, duration of PICU stay, body temperature at admission, intravenous immunoglobulin usage, red blood cell (RBC) transfusion, and lactate concentration at admission Table 2 presents the comparison of prognostic factors in PICU according to culture-positive bacterial infections. This table was created considering that culture-positive bacterial infections may affect prognostic factors. In addition, we used the control group who were blood culture negative patients in this study.

Logistic regression analysis revealed that culture-positive bacterial infections in the PICU were significantly associated with IMV use (odds ratio [OR]: 2.254, 95\% confidence interval [CI]: 1.229-4.131), RBC transfusions (OR: 2.624, 95\% CI: 1.346-5.115), and inotropic drug use (OR: 2.262, 95\% CI: 1.154-3.581) (Table 3). 
Table 2. Factors associated with culture-positive bacterial infections.

\begin{tabular}{cccc}
\hline & Culture Positive (n = 90) & Culture Negative (n = 387) & P \\
\hline Sex & & & \\
Male & $49(54.4 \%)$ & $216(55.8 \%)$ & 0.814 \\
Female & $41(45.6 \%)$ & $171(44.2 \%)$ & 0.013 \\
Age (years) & $1.0(1$ month-18 years) & $2.25(1$ day-18 years) & $<0.001$ \\
IMV support & $58(64.4 \%)$ & $136(35.1 \%)$ & $<0.001$ \\
Duration of IMV support (days) & $10(1-110)$ & $3(1-50)$ & $<0.001$ \\
Inotropic drug use & $39(43.3 \%)$ & $64(16.5 \%)$ & $<0.001$ \\
Acute kidney injury & $50(55.6 \%)$ & $87(22.5 \%)$ & 0.077 \\
NIV & $31(34.4 \%)$ & $69(17.8 \%)$ & $<0.001$ \\
Continuous renal replacement therapy & $51(56.7 \%)$ & $110(28.4 \%)$ & 0.004 \\
Duration of NIV support (h) & $8(1-144)$ & $3(1-120)$ & 0.008 \\
Mortality & $13(14.4 \%)$ & $24(6.2 \%)$ & $<0.001$ \\
Duration of PICU stay (days) & $10(1-122)$ & $3(1-59)$ & 0.436 \\
Surgery & $14(15.6 \%)$ & $49(12.7 \%)$ & $<.002$ \\
Body temperature at admission $\left({ }^{\circ} \mathrm{C}\right)$ & $37.0(34.5-39.3)$ & $36.7(33.9-39.4)$ & $<0.001$ \\
Intravenous immunoglobulin use & $30(33.3 \%)$ & $25(6.5 \%)$ & 0.001 \\
Red blood cell transfusion & $60(66.7 \%)$ & $156(40.3 \%)$ & 0.244 \\
C-reactive protein $(\mathrm{mg} / \mathrm{dL})$ & $2.0(0.3-32.2)$ & $1.0(0.1-279.3)$ & 0.206 \\
Leucocyte count $\left(/ \mathrm{mm}^{3}\right)$ & $11,800(200-111,300)$ & $10,800(100-192,000)$ & 0.089 \\
Neutrophil count $\left(/ \mathrm{mm}^{3}\right)$ & $6400(0-105,200)$ & $6150(0-54,400)$ & 0.018 \\
Lactate $(\mathrm{mmol} / \mathrm{L})$ & $1.75(0.5-17.0)$ & $1.70(0.3-20.0)$ & $<0.001$ \\
\hline Total parenteral nutrition use & $40(44.4 \%)$ & $86(22.2 \%)$ & \\
\hline
\end{tabular}

Values are reported as number (percentage) or median (range). PICU, paediatric intensive care unit; IMV, invasive mechanical ventilation, $\mathrm{NIV}$, non-invasive mechanical ventilation.

Table 3. Logistic regression analysis of prognostic factors for culture-positive bacterial infections.

\begin{tabular}{cccc}
\hline & $\mathbf{P}$ & Odds Ratio & 95\% Confidence Interval \\
\hline Invasive mechanical ventilation & 0.009 & 2.254 & $1.229-4.131$ \\
Catheter use & 0.328 & 1.419 & $0.703-2.866$ \\
Surgery & 0.497 & 1.270 & $0.637-2.530$ \\
Red blood cell transfusion & 0.005 & 2.624 & $1.346-5.115$ \\
Acute kidney injury & 0.478 & 1.312 & $0.620-2.777$ \\
Previous hospitalization & 0.180 & 1.475 & $0.836-2.602$ \\
Inotropic drug use & 0.017 & 2.262 & $1.154-4.433$ \\
Mortality & 0.561 & 1.339 & $0.500-3.581$ \\
\hline
\end{tabular}

\subsection{Factors Associated with Gram-Positive and Gram-Negative Bacterial Infections in the PICU}

There was an association between gram-negative bacterial infections and IMV support $(p=0.045)$. There was also an association between gram-positive bacterial infections and catheter-related sepsis $(\mathrm{p}=0.006)($ Table 4$)$.

Table 4. Factors associated with gram-positive and gram-negative bacterial infections.

\begin{tabular}{cccc}
\hline & Gram-Negative (n = 67) & Gram-Positive (n = 23) & P \\
\hline Sex & & & \\
Male & $34(54.4 \%)$ & $14(55.8 \%)$ & 0.401 \\
Female & $33(45.6 \%)$ & $9(44.2 \%)$ & 0.139 \\
Age (years) & $1.0(1$ month-18 years) & $1.80(1$ month-17 years) & 0.045 \\
Invasive mechanical ventilation support & $50(64.4 \%)$ & $12(35.1 \%)$ & 0.468 \\
Inotropic drug use & $35(43.3 \%)$ & $10(16.5 \%)$ & 0.833 \\
Acute kidney injury & $21(55.6 \%)$ & $8(22.5 \%)$ & 0.932 \\
Continuous renal replacement therapy & $14(34.4 \%)$ & $5(17.8 \%)$ & 0.054 \\
Non-invasive mechanical ventilation & $31(56.7 \%)$ & $16(28.4 \%)$ & 0.187 \\
Mortality & $14(14.4 \%)$ & $2(6.2 \%)$ & 0.665 \\
Duration of PICU stay $($ days) & $9(1-120)$ & $12(3-122)$ & 0.588 \\
Body temperature at admission $\left({ }^{\circ} \mathrm{C}\right)$ & $37.0(34.5-39.3)$ & $37.2(35.2-38.7)$ & 0.598 \\
Intravenous immunoglobulin use & $23(33.3 \%)$ & $10(6.5 \%)$ & 0.006 \\
Catheter-related sepsis & $3(4.5 \%)$ & $5(17.8 \%)$ & 0.261 \\
Red blood cell transfusion & $48(66.7 \%)$ & $15(40.3 \%)$ & 0.433 \\
C-reactive protein $\left(\mathrm{mg}^{\prime} \mathrm{dL}\right)$ & $2.0(0.3-32.2)$ & $2.0(0.4-30.6)$ & 0.194 \\
Leucocyte count $\left(/ \mathrm{mm}^{3}\right)$ & $13,600(200-111,800)$ & $10,300(200-40,500)$ & 0.333 \\
Neutrophil count $\left(/ \mathrm{mm}^{3}\right)$ & $7200(0-105,200)$ & $5800(100-39,500)$ & \\
\hline
\end{tabular}

Values are reported as number (percentage) or median (range). PICU, paediatric intensive care unit. 


\subsection{Factors Associated with Gram-Negative CRIs}

There were associations between gram-negative CRIs and IMV support $(p=0.019)$, duration of PICU stay ( $p=0.031)$, previous hospitalization $(p=0.045)$, RBC transfusion $(p=0.007)$, total parenteral nutrition use before infection $(\mathrm{p}<0.001)$, culture results $(\mathrm{p}<0.001)$, and culture sample source $(\mathrm{p}=0.007)$. In the carbapenem-resistant bacterial infections, there were the most common Klebsiella $\operatorname{spp}(\mathrm{n}=9)$, Pseudomonas aeruginosa $(\mathrm{n}=8)$, and Acinetobacter spp. $(\mathrm{n}=6)$, respectively. Our CRIs were healthcare-associated and most of these patients had used total parenteral nutrition before the PICU admission. In the CRI infections, intravenous colistin was used with tigecycline, rifampin or nebulized colistin, after the antibiogram and culture results (Table 5).

Table 5. Factors associated with gram-negative carbapenem-resistant bacterial infections.

\begin{tabular}{|c|c|c|c|c|}
\hline & & \multicolumn{2}{|c|}{ Carbapenem-Resistant Gram-Negative } & \multirow{2}{*}{$\mathbf{P}$} \\
\hline & & Yes $(n=25)$ & No $(n=42)$ & \\
\hline \multirow{2}{*}{ Sex } & Female & $10(40.0 \%)$ & $25(59.5 \%)$ & \multirow{2}{*}{0.122} \\
\hline & Male & $15(60.0 \%)$ & $17(40.5 \%)$ & \\
\hline \multicolumn{2}{|c|}{ Age (years) } & $\begin{array}{c}1.0 \text { (2 month-18 } \\
\text { years })\end{array}$ & $\begin{array}{c}1.0 \text { (1 month-17 } \\
\text { years) }\end{array}$ & 0.289 \\
\hline \multicolumn{2}{|c|}{ Duration of IMV support (days) } & $23(92.0 \%)$ & $28(66.7 \%)$ & 0.019 \\
\hline \multicolumn{2}{|c|}{ Inotropic drug use } & $9.0(1-110)$ & $9.5(1-52)$ & 0.559 \\
\hline \multicolumn{2}{|c|}{ Inotropic drug use } & $10(40.0 \%)$ & $25(59.5 \%)$ & 0.122 \\
\hline \multicolumn{2}{|c|}{ Acute kidney injury } & $6(24.0 \%)$ & $15(35.7 \%)$ & 0.320 \\
\hline \multicolumn{2}{|c|}{ Continuous renal replacement therapy } & $3(12.0 \%)$ & $10(23.8 \%)$ & 0.237 \\
\hline \multicolumn{2}{|c|}{ NIV } & $11(44.0 \%)$ & $20(47.6 \%)$ & 0.774 \\
\hline \multicolumn{2}{|c|}{ Duration of NIV support (h) } & $48.0(2-144)$ & $6.0(1-96)$ & 0.083 \\
\hline \multicolumn{2}{|c|}{ Mortality } & $6(24.0 \%)$ & $7(16.7 \%)$ & 0.463 \\
\hline \multicolumn{2}{|c|}{ Duration of PICU stay (days) } & $11.0(1-120)$ & $7.5(1-52)$ & 0.031 \\
\hline \multicolumn{2}{|c|}{ Intravenous immunoglobulin use } & $6(24.0 \%)$ & $17(40.5 \%)$ & 0.275 \\
\hline \multicolumn{2}{|c|}{ Previous hospitalization } & $23(92.0 \%)$ & $30(71.4 \%)$ & 0.045 \\
\hline \multicolumn{2}{|c|}{ Surgery } & $3(12.0 \%)$ & $3(7.1 \%)$ & 0.501 \\
\hline \multicolumn{2}{|c|}{ Red blood cell transfusion } & $12(48.0 \%)$ & $35(83.3 \%)$ & 0.007 \\
\hline \multicolumn{2}{|c|}{ Central venous catheter use } & $19(76.0 \%)$ & $33(78.6 \%)$ & 0.807 \\
\hline \multicolumn{2}{|c|}{ Total parenteral nutrition usage before infection } & $19(76.0 \%)$ & $15(35.7 \%)$ & $<0.001$ \\
\hline \multicolumn{2}{|c|}{ Positive culture samples taken at admission } & $20(80.0 \%)$ & $36(85.7 \%)$ & 0.541 \\
\hline \multirow{4}{*}{ Culture results } & Klebsiella spp. & $9(36.0 \%)$ & $18(42.9 \%)$ & \multirow{4}{*}{$<0.001$} \\
\hline & P. aeruginosa & $8(32.0 \%)$ & $7(16.7 \%)$ & \\
\hline & Acinetobacter spp. & $6(24.0 \%)$ & $1(2.4 \%)$ & \\
\hline & Others & $2(8.0 \%)$ & $16(38.1 \%)$ & \\
\hline \multirow{6}{*}{$\begin{array}{l}\text { Culture sample source: } \\
\text { Antibiotics used for } \\
\text { salvage treatment in } \\
\text { addition to IV colistin } \\
\text { treatment }\end{array}$} & Tracheal aspirate & $17(68.0 \%)$ & $12(28.6 \%)$ & \multirow{6}{*}{0.007} \\
\hline & Blood & $6(24.0 \%)$ & $22(52.4 \%)$ & \\
\hline & Others & $2(8.0 \%)$ & $8(19.0 \%)$ & \\
\hline & Tigecycline & $9(36.0 \%)$ & - & \\
\hline & Rifampin & $11(44.0)$ & - & \\
\hline & Nebulised colistin & $23(92.0 \%)$ & - & \\
\hline
\end{tabular}

Values are reported as number (percentage) or median (range). PICU, paediatric intensive care unit; IMV, invasive mechanical ventilation, NIV, non-invasive mechanical ventilation. 
Logistic regression analysis revealed that CRIs in the PICU were significantly associated with IMV use (OR: 7.626, 95\% CI: 1.010-57.580), RBC transfusions (OR: 0.094, 95\% CI: 0.017-0.525), and total parenteral nutrition use (OR: 5.870, 95\% CI: 1.386-24.870) (Table 6).

Table 6. Prognostic factors for carbapenem-resistant bacterial infections.

\begin{tabular}{cccc}
\hline & $\mathbf{P}$ & Odds Ratio & 95\% Confidence Interval \\
\hline Invasive mechanical ventilation & 0.005 & 7.626 & $1.010-57.580$ \\
Surgery & 0.896 & 1.112 & $0.226-5.468$ \\
Red blood cell transfusion & 0.007 & 0.094 & $0.017-0.525$ \\
Acute kidney injury & 0.318 & 0.415 & $0.074-2.330$ \\
Previous hospitalization & 0.560 & 1.965 & $0.203-19.046$ \\
Inotropic drug use & 0.669 & 1.322 & $0.923-4.421$ \\
Mortality & 0.706 & 1.341 & $0.292-6.156$ \\
Total parenteral nutrition use & 0.016 & 5.870 & $1.386-24.870$ \\
\hline
\end{tabular}

\subsection{Survival Outcomes According to Culture Results}

Kaplan-Meier curves and the log-rank test revealed no significant difference in survival between patients with positive or negative culture results during their PICU stay $(p=0.945)$ (Figure 2).

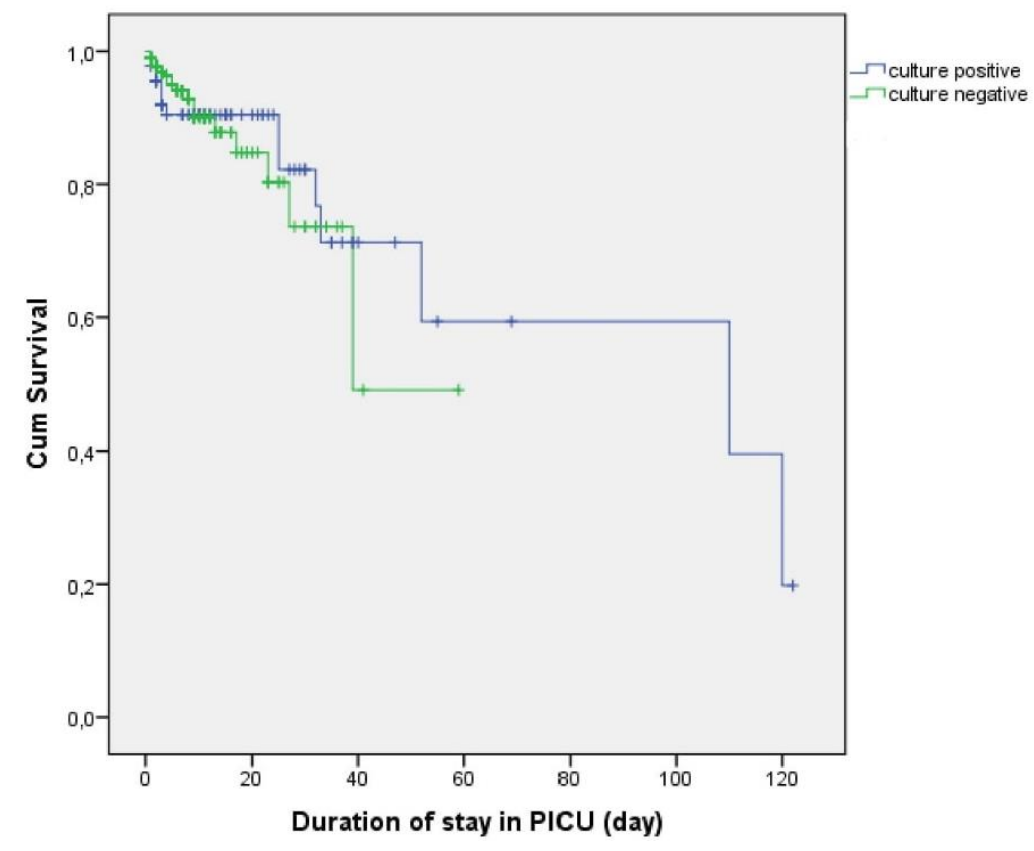

Figure 2. Kaplan-Meier curves for survival according to culture results.

However, a significant difference in survival was detected when we performed the analysis according to the culture sample source, with positive blood cultures being associated with the poorest survival, relative to the other sources ( $p=0.012$; Figure 3 ).

When we performed the analysis according to the culture sample source in the CRIs patients $(n=25)$, a significant difference in survival was detected. The positive blood cultures being associated with the poorest survival, relative to the other sources $(p=0.004$; Figure 4$)$. 


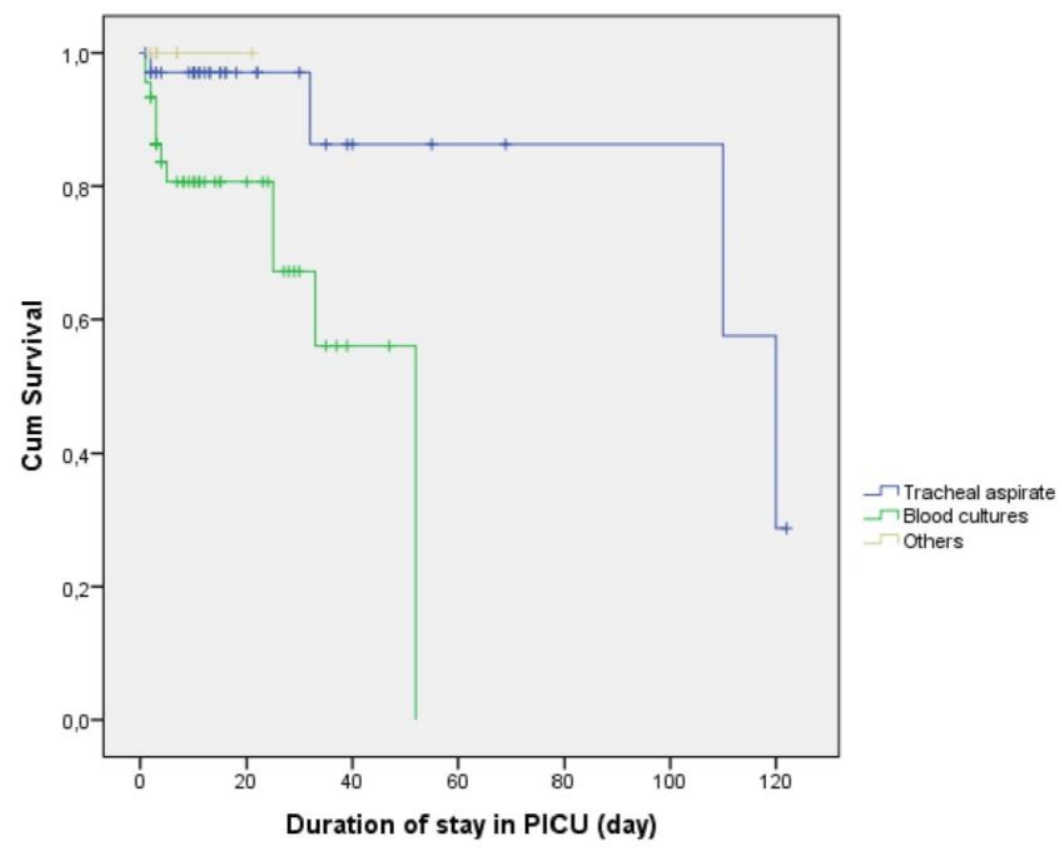

Figure 3. Kaplan-Meier curves for survival according to culture sample source.

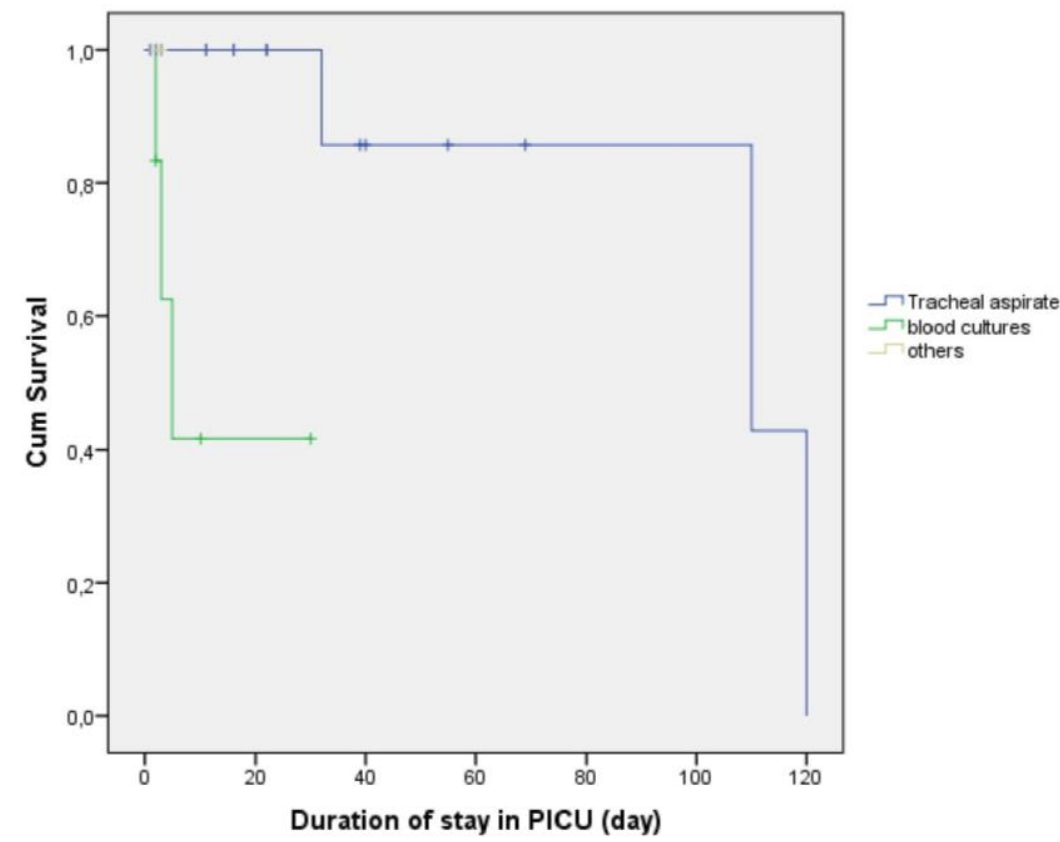

Figure 4. Kaplan-Meier curves for survival according to culture sample source in the CRIs patients $(\mathrm{p}=0.004)$.

\section{Discussion}

The present study revealed that infections in our PICU were associated with poor outcomes among critically ill children, which highlights the importance of understanding the causes of PICU infections. It highlights the importance of early detection and appropriate management. Among the 90 patients $(18.9 \%)$ with bacterial infections, the infectious agents were predominantly gram-negative bacteria, although mortality was not associated with gram-negative or gram-positive status. However, patients with positive culture results were younger than patients with negative culture results, and age was associated with mortality and various clinical factors. Cases with CRIs were frequently detected using tracheal aspirate samples and frequently involved mechanical ventilator use, with the infectious agents 
typically being Klebsiella spp. and Pseudomonas spp. Our CRIs were healthcare-associated and most of these infected patients had used total parenteral nutrition before the PICU admission. Positive blood cultures were associated with poorer survival than positive tracheal aspirate cultures, and no deaths were observed in cases with isolated positive results from urine or cerebrospinal fluid cultures.

Most infections in our study involved gram-negative bacteria, and recent studies have focused on the treatment and prevention of gram-negative bacterial infections, given the rise in drug resistance [7-10]. Many mechanisms were described about the development of carbapenem resistance. Multidrug efflux pumps are very important in this setting [11-13]. This mechanism may be related to the increasing rates of CRIs, which can also involve multidrug resistance $[14,15]$. Recently, we observed that the frequency of CRIs increased in our PICU. Therefore, we have planned this study. Carbapenem resistance was identified in approximately one-third of gram-negative culture results, with $80 \%$ of these infections involving paediatric ward patients. The present study revealed CRIs in 25 patients, with 20 of these cases detected at the PICU admission. Most cases involved positive tracheal aspirate cultures and the most important risk factor for CRIs was previous hospitalization.

In 23 patients, we used tigecycline, rifampin, and nebulised colistin for salvage treatment in addition to IV colistin treatment. All these drugs, we used in various combinations with other antibiotics. CRIs have the potential to develop resistance during single-agent therapy. In the literature, tigecycline monotherapy used in the multidrug-resistant Gram-negative bacilli and tigecycline resistance had reported $[16,17]$. In a recent study showed that triple or double combination of meropenem, colistin and tigecycline were bactericidal against carbapenem-resistant Klebsiella pneumoniae isolates [18]. In another study, it was reported that combination antibiotic therapy including colistin and tigecycline may have better ability in preventing the evolution of resistance in CRIs [19]. However, another two studies reported that resistant strains were isolated during the combination therapy of colistin and tigecycline $[20,21]$. We think that combined colistin and tigecycline treatment provided synergistic effect against CRIs, like the first studies. The mortality of colistin-tigecycline combination treatment was limited to only 1 patient. However, there were 9 patients who used tigecycline-colistin combination treatment usage only. Rifampicin-based regimens have been shown to be effective against carbapenem-resistant $A$. baumannii infection. Because it was showed colistin-rifampicin combinations inhibit producing the bacterial biofilm [22]. Therefore, we used rifampicin all of the carbapenem-resistant Acinetobacter spp.

Intensive care units are the most common location for nosocomial infections because of invasive procedures $[2,3,8,10]$. In developed countries, invasive device-associated bloodstream infections and respiratory infections are the most common types of nosocomial infections in the PICU $[3,10]$. For example, ventilator-associated pneumonia and nosocomial infections are more common in patients with mechanical ventilation use $[23,24]$. The clearest risk factor for these infections is reportedly the duration of IMV usage [25]. In the present study, our nosocomial infection rate was low, although most infections were detected at admission and infections were predominantly detected using tracheal aspirate samples. Logistic regression analysis revealed that IMV use was associated with a 2.25-fold higher likelihood of infection, although prolonged IMV use was not associated with an increased likelihood of infection.

Another risk factor for infections in the PICU is vascular catheter use [26], and it is important to identify and manage related risk factors, as catheter-related infections have high mortality and morbidity rates [27]. These infections most commonly involve gram-positive cocci (coagulase-negative staphylococci, such as S. aureus) [28]. However, the present study failed to detect significant differences in infection rates according to catheter type or location, and the logistic regression analysis revealed that catheter use was not associated with an increased infection rate. This may be related to our centre's adoption of a "central line bundle" strategy for preventing infections, which was developed based on the existing literature [29]. The most common catheter-related bacterial infections involved gram-positive bacteria in our PICU, which agrees with previous findings [28]. However, a positive 
blood culture result for S. epidermidis does not necessarily confirm an infection, therefore we used other infection markers and clinical findings to confirm the same.

Blood component transfusions are also reportedly a risk factor for infection [30], and can increase the risk of mortality through immunosuppression and the increased risk of nosocomial infection [31]. The present study revealed that culture-positive cases had an increased need for RBC transfusions (2.6-fold more likely in culture-positive cases), although RBC transfusions were not associated with gram-negative or gram-positive culture results. Interestingly, greater RBC transfusion use was associated with a lower rate of CRIs. However, we could not explain this difference.

Among critically ill patients, AKI is an independent predictor of a poor prognosis [32]. This may be related to AKI being associated with an increased risk of infection, which is related to uremic retention and metabolic acidosis having negative effects on immunity [33]. Exposure to catheters and other invasive therapies, such as CRRT, may also increase the risk of infection. For example, the rates of infection and sepsis were higher in adult patients with AKI treated using CRRT than patients treated without CRRT [34]. Santiago et al. also recently reported a high infection rate among critically ill children treated using CRRT, especially in cases with $>4$ days of CRRT use [35]. The present study revealed that AKI was associated with a higher infection rate, although CRRT use was not associated with infection risk. Most patients who required CRRT had infections that were detected at admission, and the logistic regression analysis revealed no relationships between culture-positive infections and AKI or CRRT.

Akturk et al. recently reported that previous surgery was an independent risk factor for carbapenem-resistant Klebsiella infection in the PICU [10]. In contrast, the present study predominantly identified infections using tracheal aspirate samples, and previous surgery ( $92.0 \%$ of patients) was not associated with the risk of infection. Gram-negative CRIs were associated with a mortality rate of $24 \%$ and gram-negative CRIs did not have a significantly higher mortality rate. However, CRIs were associated with IMV use and prolonged hospitalization, with the logistic regression analysis revealing that CRIs were associated with a 7.626-fold higher likelihood of IMV use. The present study also revealed that CRIs were most commonly detected in cases with total parenteral nutrition use. This relationship may be related to many PICU patients who received total parenteral nutrition before their admission, had concomitant chronic diseases, and/or had a previous hospitalization. The most common patients with CRIs came from the metabolism, infection and gastroenterology wards. This situation may have been caused by frequent use of total parenteral nutrition in these wards.

Previous reports have discussed the relationship between inotropic drug use and prognosis, although the results are conflicting. For example, one study revealed that delaying inotropic treatment was associated with increased mortality [36], while another study revealed that inotropic drug use was associated with a poor prognosis and mortality [37]. Therefore, we evaluated the relevance of inotropic drug use in our PICU, and found that culture positivity was associated with a 2.2620-fold higher likelihood of inotropic drug use.

Most of our patients with positive culture results at their PICU admission had gram-negative bacterial infections. Our CRIs were healthcare-associated and most of these patients had used antibiotics before the PICU admission. Therefore, the patients with positive culture results at admission had significantly longer PICU stays, because of longer treatment time. Nosocomial infections were also associated with prolonged PICU stays, which agrees with the findings of Dominguez et al. [38].

Bloodstream infections are a major cause of morbidity and mortality in critically ill patients. Our survival analysis revealed that positive blood culture results were associated with poorer survival than positive tracheal aspirate culture results. In a recent study showed that mortality of bloodstream infections was high and having resistant bacteria were independently associated with 30-day mortality [39]. Similarly, higher mortality was found in CRI related bloodstream infections in our study (Figure 4). Three of 6 patients with CRI related bloodstream infections died and all of them had Klebsiella pneumoniae. They died within 2,3, and 5 days respectively. Another common point in these patients, they were admitted to the intensive care unit with the diagnosis of septic shock. 
The positive CRI in tracheal aspirate were 17 patients and three of them were died. In the tracheal aspirate cultures, two of these patients had A. baumannii and one had Pseudomonas spp. They were died in 32, 110 and 120 days respectively. Another common point in these three patients was that they had a previous history of intubation and PICU stay. In contrast, all patients with isolated positive results from urine or cerebral spinal fluid cultures survived and had relatively short PICU stays.

The present study's findings are limited by the retrospective single-centre design, low number of patients, and heterogeneous populations (different ages). In addition, it is associated with risks of bias and the possibility that different results may be observed in other centres.

\section{Materials and Methods}

\subsection{Study Design}

PICU at Istanbul University-Cerrahpaşa has 7 beds, 7 ventilators, and 2 isolation rooms for treating children who are 1 day to 18 years old. For invasive procedures such as CRRT and extracorporeal membrane oxygenation (ECMO), newborns were rarely admitted to the PICU. Electronic and medical records were retrospectively searched to collect data regarding the 477 patients who were admitted to the PICU for various critical illnesses between January 2014 and March 2019. We excluded patients with a PICU stay duration of $<24 \mathrm{~h}$, patients who died during the first day after admission, and patients with fungal and multi-bacterial culture results. The study was conducted in accordance with the Declaration of Helsinki. The study's retrospective protocol was approved by the institutional ethics committee (İstanbul University-Cerrahpaşa, 29430533-903.99-184025, 21 May 2018), and all study-related anonymized data are available upon reasonable request.

\subsection{Data Collection and Patient Comparisons}

The study variables included demographic and clinical factors, such as sex, age, invasive or non-invasive mechanical ventilation use, duration of PICU stay, mortality, Pediatric Risk of Mortality Score (PRISM) III score, CRRT use, haemogram findings, biochemistry results, and blood gases at admission. Patients were compared according to whether they had positive or negative culture findings, gram-negative or gram-positive infections, and infections that did or did not involve carbapenem resistance.

We defined AKI as oliguria (urine output of $<0.5 \mathrm{~mL} / \mathrm{kg} / \mathrm{h}$ ) and an elevated serum creatinine value for the patient's age or a 1.5-fold increase in the creatinine concentration at $24 \mathrm{~h}$ (relative to admission). The estimated glomerular filtration rate was calculated according to the original Schwartz formula, based on serum urea and creatinine concentrations that were measured using standard laboratory procedures.

The PRISM III score was calculated via a tool available on the Turkish Ministry of Health website (https://kalite.saglik.gov.tr/). This tool calculates the PRISM score based on the values for the lowest/highest systolic blood pressures from the first $24 \mathrm{~h}$, diastolic blood pressure, heart rate, respiratory rate, $\mathrm{PaO}_{2} / \mathrm{FiO}_{2}, \mathrm{PaCO}_{2}$, prothrombin time/partial thromboplastin time, serum total bilirubin, calcium, potassium, glucose, bicarbonate, pupillary response, and Glasgow coma score.

\subsection{Evaluation of Culture Results}

A single blood culture was performed for each case to identify any known pathogens. In cases with a positive result, confirmation of infection was based on a second positive blood culture or detection of the same pathogen in separate peripheral and central blood cultures [2]. Tracheal aspirate samples were used to diagnose pneumonia in cases with a count of $>10^{3}$ colony-forming units/mL [3]. Urine cultures were considered positive in cases with a count of $>10^{5}$ colony-forming units $/ \mathrm{mL}$ [4]. 


\subsection{Statistical Analysis}

All statistical analyses were performed using IBM SPSS software (version 21.0; IBM Corp., Armonk, NY, USA). Continuous data were expressed as median (range) and categorical data were expressed as number (percentage). Continuous variables were compared using Student's t-test for parametric data and the Mann-Whitney U-test for non-parametric data. Categorical variables were compared using the chi-squared test or Fisher's exact test. Univariate binary logistic regression models were used to calculate the relationships between risk factors and CRIs infections, culture positive infections with the results reported as ORs and 95\% CIs. Survival data were calculated using Kaplan-Meier Log-Rank statistical analysis for cultures results and culture sample source. Differences were considered statistically significant at $\mathrm{p}$-values of $<0.05$.

\section{Conclusions}

The present study revealed that infections, especially CRIs, were associated with poor outcomes among critically ill children in the PICU. In addition, infections in the PICU were associated with increased use of IMV, RBC transfusions, and inotropic drugs. Furthermore, carbapenem resistance was found in approximately one-third of gram-negative culture results, and total parenteral nutrition was a risk factor for developing CRIs. We conclude that CRIs are a serious issue among critically ill children, and that reducing the inappropriate use of antibiotics, especially carbapenems, will be important in preventing multidrug-resistant bacterial infections.

Author Contributions: Conceptualization, F.A.; Data curation, F.A., F.D.A., F.V. and C.D.; Formal analysis, F.A.; Funding acquisition, F.A.; Investigation, F.A.; Methodology, F.A.; Project administration, F.A.; Resources, F.A., F.V. and C.D.; Software, F.A.; Supervision, F.A., H.Ç. (Haluk Çokuğraş), Y.C. and H.Ç. (Halit Çam); Validation, F.A. and C.D.; Visualization, F.A. and F.V.; Writing-original draft, F.A.; Writing review \& editing, F.A., F.D.A., H.Ç. (Haluk Çokuğraş), Y.C., and H.Ç (Halit Çam).

Funding: This research received no external funding.

Conflicts of Interest: The authors declare no conflict of interest.

\section{References}

1. Dorofaeff, T.; Mohseni-Bod, H.; Cox, P.N. Infections in the PICU. In Textbook of Clinical Pediatrics; Elzouki, A.Y., Harfi, H.A., Nazer, H.M., Stapleton, B., Oh, W., Eds.; Springer: Berlin/Heidelberg, Germany, 2012; pp. 2537-2563.

2. Esteban, E.; Ferrer, R.; Urrea, M.; Suarez, D.; Rozas, L.; Balaguer, M. The impact of a quality improvement intervention to reduce nosocomial infections in a PICU. Pediatr. Crit. Care Med. 2013, 14, 525-532. [CrossRef] [PubMed]

3. Grohskopf, L.A.; Sinkowitz-Cochran, R.L.; Garrett, D.O.; Sohn, A.H.; Levine, G.L.; Siegel, J.D.; Stover, B.H.; Jarvis, W.R.; Pediatric Prevention Network. A national point-prevalence survey of pediatric intensive care unit-acquired infections in the United States. J. Pediatr. 2002, 140, 432-438. [CrossRef]

4. Reymond, J.; Aujard, Y.; European Study Group. Nosocomial infections in pediatric patients: A European multicenter prospective study. Infect. Control Hosp. Epidemiol. 2000, 21, 260-263. [CrossRef]

5. Dray, S.; Coiffard, B.; Persico, N.; Papazian, L.; Hraiech, S. Are tracheal surveillance cultures useful in the intensive care unit? Ann. Transl. Med. 2018, 6, 421. [CrossRef]

6. Karageorgos, S.A.; Bassiri, H.; Siakallis, G.; Miligkos, M.; Tsioutis, C. Intravenous colistin use for infections due to MDR Gram-negative bacilli in critically ill paediatric patients: A systematic review and meta-analysis. J. Antimicrob. Chemother. 2019. [CrossRef]

7. Stein, R.; Dogan, H.S.; Hoebeke, P.; Kočvara, R.; Nijman, R.J.M.; Radmayr, C.; Tekgül, S.; European Association of Urology; European Society for Pediatric Urology. Urinary tract infection in children: EAU/ESPU guidelines. Eur. Urol. 2015, 67, 546-558.

8. El-Nawawy, A.; Ashraf, G.A.; Antonios, M.A.M.; Meheissen, M.A.; El-Alfy, M.M.R. Incidence of Multidrug-Resistant Organism Among Children Admitted to Pediatric Intensive Care Unit in a Developing Country. Microb. Drug Resist. 2018, 24, 1198-1206. [CrossRef] [PubMed] 
9. Akturk, H.; Sutcu, M.; Somer, A.; Aydın, D.; Cihan, R.; Ozdemir, A. Carbapenem-resistant Klebsiella pneumoniae colonization in pediatric and neonatal intensive care units: Risk factors for progression to infection. Braz. J. Infect. Dis. 2016, 20, 134-140. [CrossRef]

10. Aygun, F.; Aygun, F.D.; Varol, F.; Durak, C.; Cokugraş, H.; Camcioglu, Y.; Cam, H. Can Nebulised Colistin Therapy Improve Outcomes in Critically Ill Children with Multi-Drug Resistant Gram-Negative Bacterial Pneumonia? Antibiotics 2019, 8, 40. [CrossRef]

11. Nikaido, H.; Pagès, J.M. Broad-specificity efflux pumps and their role in multidrug resistance of Gram-negative bacteria. FEMS Microbiol. Rev. 2012, 36, 340-363. [CrossRef] [PubMed]

12. Pagès, J.M.; Amaral, L. Mechanisms of drug efflux and strategies to combat them: Challenging the efflux pump of Gram-negative bacteria. Biochim. Biophys. Acta 2009, 1794, 826-833. [CrossRef]

13. Pan, Y.P.; Xu, Y.H.; Wang, Z.X.; Fang, Y.P.; Shen, J.L. Overexpression of MexAB-OprM efflux pump in carbapenem-resistant Pseudomonas aeruginosa. Arch. Microbiol. 2016, 198, 565-571. [CrossRef]

14. Li, J.; Nation, R.L.; Milne, R.W.; Turnidge, J.D.; Coulthard, K. Evaluation of colistin as an agent against multiresistant Gram-negative bacteria. Int. J. Antimicrob. Agents 2005, 25, 11-25. [CrossRef]

15. Falagas, M.E.; Kasiakou, S.K. Colistin: The revival of polymyxins for the management of multidrug resistant gram-negative bacterial infections. Clin. Infect. Dis. 2005, 40, 1333-1341. [CrossRef]

16. Anthony, K.B.; Fishman, N.O.; Linkin, D.R.; Gasink, L.B.; Edelstein, P.H.; Lautenbach, E. Clinical and microbiological outcomes of serious infections with multidrug-resistant gram-negative organisms treated with tigecycline. Clin. Infect. Dis. 2008, 46, 567-570. [CrossRef] [PubMed]

17. Ye, J.J.; Lin, H.S.; Kuo, A.J.; Leu, H.S.; Chiang, P.C.; Huang, C.T.; Lee, M.H. The clinical implication and prognostic predictors of tigecycline treatment for pneumonia involving multidrug-resistant Acinetobacter baumannii. J. Infect. 2011, 63, 351-361. [CrossRef]

18. Tsala, M.; Vourli, S.; Georgiou, P.C.; Pournaras, S.; Daikos, G.R.L.; Mouton, J.W.; Meletiadis, J. Triple combination of meropenem, colistin and tigecycline was bactericidal in a dynamic model despite mere additive interactions in chequerboard assays against carbapenemase-producing Klebsiella pneumoniae isolates. J. Antimicrob. Chemother. 2019, 74, 387-394. [CrossRef]

19. Ni, W.; Wei, C.; Zhou, C.; Zhao, J.; Liang, B.; Cui, J.; Wang, R.; Liu, Y. Tigecycline-Amikacin Combination Effectively Suppresses the Selection of Resistance in Clinical Isolates of KPC-Producing Klebsiella pneumoniae. Front. Microbiol. 2016, 7, 1304. [CrossRef]

20. Elemam, A.; Rahimian, J.; Mandell, W. Infection with panresistant Klebsiella pneumoniae: A report of 2 cases and a brief review of the literature. Clin. Infect. Dis. 2009, 49, 271-274. [CrossRef]

21. Cho, S.Y.; Kang, C.I.; Chung, D.R.; Peck, K.R.; Song, J.H.; Jang, J.H. Breakthrough bacteremia due to extended-spectrum-beta lactamase-producing Klebsiella pneumoniae during combination therapy with colistin and tigecycline. Antimicrob. Agents Chemother. 2012, 56, 4994-4995. [CrossRef]

22. Song, J.Y.; Lee, J.; Heo, J.Y.; Noh, J.Y.; Kim, W.J.; Cheong, H.J.; Hwang, I.S. Colistin and rifampicin combination in the treatment of ventilator-associated pneumonia caused by carbapenem-resistant Acinetobacter baumannii. Int. J. Antimicrob. Agents 2008, 32, 281-284. [CrossRef]

23. Majumdar, S.S.; Padiglione, A.A. Nosocomial infections in the intensive care unit. Anaesthasia Intensiv. Care Med. 2012, 13, 204-208. [CrossRef]

24. Gupta, A.; Kapil, A.; Lodha, R.; Kabra, S.K.; Sood, S.; Dhawan, B.; Das, B.K.; Sreenivas, V. Burden of healthcare-associated infections in a paediatric intensive care unit of a developing country: A single centre experience using active surveillance. J. Hosp. Infect. 2011, 78, 323-326. [CrossRef]

25. Venier, A.G.; Gruson, D.; Lavigne, T.; Jarno, P.; L’hériteau, F.; Coignard, B.; Savey, A.; Rogues, A.M.; REA-RAISIN Group. Identifying new risk factors for Pseudomonas aeruginosa pneumonia in intensive care units: Experience of the French national surveillance, REA-RAISIN. J. Hosp. Infect. 2011, 79, 44-48. [CrossRef]

26. Torre, F.P.F.; Baldanzi, G.; Troster, E.J. Risk factors for vascular catheter-related bloodstream infections in pediatric intensive care units. Revista Brasileira de terapia intensiva 2018, 30, 436-442. [CrossRef]

27. Rosenthal, V.D.; Guzman, S.; Migone, O.; Crnich, C.J. The attributable cost, length of hospital stay, and mortality of central line-associated bloodstream infection in intensive care departments in Argentina A prospective, matched analysis. Am. J. Infect. Control 2003, 31, 475-480. [CrossRef]

28. Wylie, M.C.; Graham, D.A.; Potter-Bynoe, G.; Kleinman, M.E.; Randolph, A.G.; Costello, J.M.; Sandora, T.J. Risk factors for central line-associated bloodstream infection in pediatric intensive care units. Infect. Control Hosp. Epidemiol. 2010, 31, 1049-1056. [CrossRef] 
29. O'Grady, N.P.; Alexander, M.; Burns, L.A.; Dellinger, E.P.; Garland, J.; Heard, S.O.; Lipsett, P.A.; Masur, H.; Mermel, L.A.; Pearson, M.L.; et al. Guidelines for the prevention of intravascular catheter-related infections. Clin. Infect. Dis. 2011, 52, e162-e193. [CrossRef]

30. Dellinger, E.P.; Anaya, D.A. Infectious and immunologic consequences of blood transfusion. Crit. Care 2004, 8, S18. [CrossRef]

31. Muszynski, J.A.; Spinella, P.C.; Cholette, J.M.; Acker, J.P.; Hall, M.W.; Juffermans, N.P.; Kelly, D.P.; Blumberg, N.; Nicol, K.; Liedel, J.; et al. Transfusion-related immunomodulation: Review of the literature and implications for pediatric critical illness. Transfusion 2017, 57, 195-206. [CrossRef]

32. Sanchez-Pinto, L.N.; Goldstein, S.L.; Schneider, J.B.; Khemani, R.G. Association between Progression and Improvement of Acute Kidney Injury and Mortality in Critically Ill Children. Pediatr. Crit. Care Med. 2015, 16, 703-710. [CrossRef]

33. Bajwa, A.; Kinsey, G.R.; Okusa, M.D. Immune mechanisms and novel pharmacological therapies of acute kidney injury. Curr. Drug Targets 2009, 10, 1196-1204. [CrossRef]

34. Thakar, C.V.; Yared, J.P.; Worley, S.; Cotman, K.; Paganini, E.P. Renal dysfunction and serious infections after open-heart surgery. Kidney Int. 2003, 64, 239-246. [CrossRef]

35. Santiago, M.J.; López-Herce, J.; Urbano, J.; Solana, M.J.; del Castillo, J.; Ballestero, Y.; Botrán, M.; Bellón, J.M. Clinical course and mortality risk factors in critically ill children requiring continuous renal replacement therapy. Intensiv. Care Med. 2010, 36, 843-849. [CrossRef]

36. Belletti, A.; Castro, M.L.; Silvetti, S.; Greco, T.; Biondi-Zoccai, G.; Pasin, L.; Zangrillo, A.; Landoni, G. The Effect of inotropes and vasopressors on mortality: A meta-analysis of randomized clinical trials. Br. J. Anaesth. 2015, 115, 656-675. [CrossRef]

37. Benbenishty, J.; Weissman, C.; Sprung, C.L.; Brodsky-Israeli, M.; Weiss, Y. Characteristics of patients receiving vasopressors. Heart Lung 2011, 40, 247-252. [CrossRef]

38. Dominguez, T.E.; Chalom, R.; Costarino, A.T., Jr. The impact of adverse patient occurrences on hospital costs in the pediatric intensive care unit. Crit. Care Med. 2001, 29, 169-174. [CrossRef]

39. Hattori, H.; Maeda, M.; Nagatomo, Y.; Takuma, T.; Niki, Y.; Naito, Y.; Sasaki, T.; Ishino, K. Epidemiology and risk factors for mortality in bloodstream infections: A single-center retrospective study in Japan. Am. J. Infect. Control 2018, 46, e75-e79. [CrossRef]

(C) 2019 by the authors. Licensee MDPI, Basel, Switzerland. This article is an open access article distributed under the terms and conditions of the Creative Commons Attribution (CC BY) license (http://creativecommons.org/licenses/by/4.0/). 